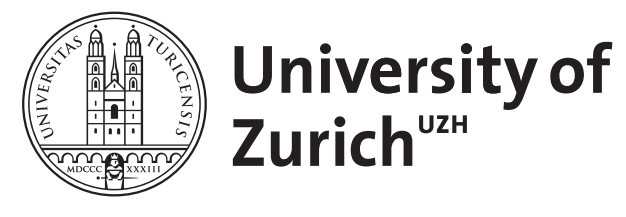

\title{
Schlüssel zur Diagnostik in der Pneumologie
}

Speich, R

\begin{abstract}
Zusammenfassung: Im Zeitalter der hoch technisierten Medizin werden Anamnese und klinische Untersuchung zunehmend in den Hintergrund gedrängt. Es ist aber eine Tatsache, dass damit auch heute noch $80 \%$ aller Diagnosen gestellt werden können. Falls überhaupt notwendig, dienen weitere, zumeist apparative Tests nur noch der Bestätigung der Diagnose. Eine kompetente Anamnese und klinische Untersuchung machen nicht selten teure und/oder zeitaufwendige Explorationen unnötig. Vor allem gilt es zu betonen, dass die aufgrund des klinischen Eindrucks gewonnene subjektive Einschätzung der Vortest-Wahrscheinlichkeit für das Vorliegen einer Krankheit eine unabdingbare Voraussetzung für die Bewertung weiterer Testresultate ist
\end{abstract}

DOI: https://doi.org/10.1007/s10405-011-0561-z

Posted at the Zurich Open Repository and Archive, University of Zurich

ZORA URL: https://doi.org/10.5167/uzh-156711

Journal Article

Published Version

Originally published at:

Speich, R (2012). Schlüssel zur Diagnostik in der Pneumologie. Der Pneumologe, 9(1):63-80.

DOI: https://doi.org/10.1007/s10405-011-0561-Z 
Pneumologe 2012 · 9:63-80

DOI 10.1007/s10405-011-0561-z

c) Springer-Verlag 2012

\section{Redaktion}

H. Olschewski, Graz

M. Rolke, Aschaffenburg

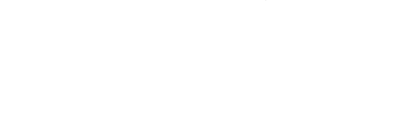

\section{Punkte sammeln auf...}

\section{CME.springer.de}

\section{Teilnahmemöglichkeiten}

- kostenfrei im Rahmen des jeweiligen Zeitschriftenabonnements

- individuelle Teilnahme durch den Erwerb

von CME.Tickets auf CME.springer.de

\section{Zertifizierung}

Diese Fortbildungseinheit ist mit 3 CME-Punkten zertifiziert von der Landesärztekammer Hessen und der Nordrheinischen Akademie für Ärztliche Fort- und Weiterbildung und damit auch für andere Ärztekammern anerkennungsfähig.

\section{Hinweis für Leser aus Österreich} Gemäß dem Diplom-FortbildungsProgramm (DFP) der Österreichischen Ärztekammer werden die auf CME. springer.de erworbenen CME-Punkte hierfür 1:1 als fachspezifische Fortbildung anerkannt.

\section{Kontakt und weitere \\ Informationen}

Springer-Verlag GmbH

Fachzeitschriften Medizin/Psychologie

CME-Helpdesk, Tiergartenstraße 17

69121 Heidelberg

E-Mail:cme@springer.com

\section{CME Weiterbildung Zertifizierte Fortbildung}

\section{R. Speich}

Universitätsspital Zürich, Zürich

\section{Schlüssel zur Diagnostik in der Pneumologie}

\section{Anamnese und klinische Untersuchung}

\section{Zusammenfassung}

Im Zeitalter der hoch technisierten Medizin werden Anamnese und klinische Untersuchung zunehmend in den Hintergrund gedrängt. Es ist aber eine Tatsache, dass damit auch heute noch $80 \%$ aller Diagnosen gestellt werden können. Falls überhaupt notwendig, dienen weitere, zumeist apparative Tests nur noch der Bestätigung der Diagnose. Eine kompetente Anamnese und klinische Untersuchung machen nicht selten teure und/oder zeitaufwendige Explorationen unnötig. Vor allem gilt es zu betonen, dass die aufgrund des klinischen Eindrucks gewonnene subjektive Einschätzung der Vortest-Wahrscheinlichkeit für das Vorliegen einer Krankheit eine unabdingbare Voraussetzung für die Bewertung weiterer Testresultate ist.

\section{Schlüisselwörter}

Lunge · Anamnese $\cdot$ Klinische Untersuchung · Lebensqualität · Evidenzbasierte Medizin 
Nach Lektüre dieses Beitrags werden Sie die nachfolgenden Punkte bewusster in Ihre Alltagspraxis einbeziehen:

- Im Zeitalter der hoch technisierten Medizin werden Anamnese und klinische Untersuchung zunehmend in den Hintergrund gedrängt [1].

- Anamnese und klinische Untersuchung erlauben auch heute noch in $80 \%$ der Fälle eine richtige Diagnosestellung. Apparative Tests dienen dann einzig der Bestätigung der Verdachtsdiagnose.

- Nur selten sind apparative Tests die einzige Möglichkeit zur Feststellung einer Diagnose.

- Aufgrund des Bayes-Wahrscheinlichkeitstheorems ist die persönliche, subjektiv geschätzte Vortest-Wahrscheinlichkeit unabdingbar in der Bewertung nachfolgender apparativer Tests. Diese probabilistische Sichtweise lässt sich leicht umsetzen, indem man anstelle von Vortest-Wahrscheinlichkeiten (wobei es nota bene nicht um einzelne

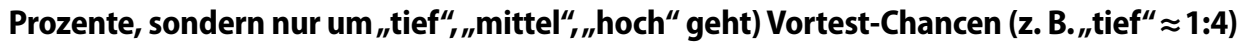
beurteilt und diese dann mit den Likelihood-Ratios, die meistens aus der Literatur bekannt sind, multipliziert, um so auf die Chance für das Vorliegen einer Krankheit nach Durchführung eines apparativen Tests zu kommen.

- Anamnese und klinische Untersuchung sind von größter Bedeutung zum Aufbau eines für die weitere Betreuung wichtigen Vertrauensverhältnisses mit den Patienten.

\section{Anamnese}

\section{Dyspnoe}

Als Dyspnoe wird eine als unangenehm empfundene Atmung bezeichnet. Als Erstes sollte eine akute Dyspnoe (auftretend innerhalb Minuten bis Stunden) von einer chronischen Dyspnoe (sich schleichend entwickelnd und persistierend) abgegrenzt werden. Die Differenzialdiagnosen und das klinische Vorgehen sind bei den beiden Formen grundsätzlich verschieden. Die wichtigsten Ursachen einer akuten Dyspnoe sind: Asthmaanfall, COPD-Exazerbation, Lungenödem, Lungenembolie, Pneumonie, Pneumothorax, Obstruktion der oberen Atemwege sowie Panikattacke („Hyperventilation“). Erwähnt sei auch die Dyspnoe im Rahmen einer „großen“ Kussmaul-Atmung bei Azidose im Rahmen eines Diabetes oder auch einer renalen Erkrankung. Auch Obstruktionen im Bereich der oberen Luftwege müssen der Vollständigkeit halber erwähnt werden, wie etwa eine ,vocal cord dysfunction“ (VCD), einseitige oder insbesondere beiderseitige Rekurrensparese, Reinke-Ödem und letztlich auch Tumoren im Bereich der oberen Atemwege. Auf die Differenzialdiagnose der kardialen Dyspnoe wird hier verzichtet.

Es muss betont werden, dass in dieser Situation heutzutage die Messung der forcierten exspiratorischen Vitalkapazität $(\mathrm{FVC})$ und des Erstsekundenvolumens $\left(\mathrm{FEV}_{1}\right)$ mittels eines Mikrospirometers obligatorisch ist. Auch die Bestimmung des Peak Flows kann zur

\section{The key to diagnosis in respiratory medicine . History taking and clinical examination}

\author{
Abstract \\ In times of high-tech medicine, history taking and physical examination are often neglected. It is, \\ however, a fact that even nowadays the patient's history and clinical findings enable a firm diagno- \\ sis to be made in $80 \%$ of the cases. If at all necessary, further technical methods just serve as a confir- \\ mation of the suspected diagnosis. Not infrequently, a competent and comprehensive clinical evalu- \\ ation renders further expensive and/or time-consuming tests unnecessary. In particular, it has to be \\ underscored that the subjective pretest probability obtained by history taking and physical examina- \\ tion is the mainstay for the interpretation of further test results. \\ Keywords \\ Pulmonary · History taking · Physical examination · Quality of life · Evidence-based medicine
}


Differenzialdiagnose COPD vs. Herzinsuffizienz verwendet werden. Routinemäßig wird immer die Sauerstoffsättigung perkutan mittels Pulsoximeter geprüft. Dabei ist aber wichtig zu wissen, dass eine zusätzliche Blutgasanalyse unabdingbar ist, da nur so das $\mathrm{p}_{\mathrm{a}} \mathrm{CO}_{2}$ und somit die alveoloarterielle Sauerstoffpartialdruckdifferenz sowie die Parameter $\mathrm{pH}$ (und somit der Basenexzess) erfasst werden können.

Bei einer chronischen Dyspnoe unterteilt man praktisch am einfachsten 3 Gruppen:

- Herzkrankheiten (Herzinsuffizienz),

- Lungenkrankheiten (obstruktive und restriktive Ventilationsstörungen) und

— anderes (pulmonale Hypertonie, neuromuskuläre Erkrankungen, Anämie und Hyperthyreose).

Zur Einteilung des Schweregrades einer Dyspnoe wird in der pneumologischen Literatur oft die Medical Research Council (MRC)-Dyspnoe-Skala verwendet. Diese ist aber wenig bekannt und im Wesentlichen vergleichbar mit der ursprünglich für Herzkrankheiten entwickelten NYHAKlassifikation. Die MRC-Skala hat 5 Grade, wobei Grad 1 „keine Dyspnoe“ in der NYHAKlassifikation nicht vorkommt. Bei der pulmonalen Hypertonie wurde leider noch eine weitere Bezeichnung „WHO-Klassifikation“ eingeführt, die aber mit der NYHA-Einteilung fast identisch ist.

Die offizielle NYHA-Klassifikation unterscheidet

- Klasse 1 (keine Dyspnoe bei alltäglichen Belastungen),

- Klasse 2 (Dyspnoe bei alltäglicher Belastung),

- Klasse 3 (Dyspnoe bereits bei leichter Belastung) und

- Klasse 4 (Dyspnoe bei allen körperlichen Aktivitäten oder sogar in Ruhe).

Werden aber diese Fragen ungezielt verwendet, ist die Korrelation zwischen verschiedenen Untersuchern gerade mal 0,5, was einer Entscheidung mittels Münzwurf entspricht. Obwohl es noch keine validierten Fragebögen gibt, sollte man sich konkrete Fragen nach dem Auftreten der Dyspnoe zurechtlegen, wie z. B.:

- Klasse 1: nur bei starken körperlichen Belastungen,

- Klasse 2: beim Treppensteigen von 2 Stockwerken und mehr in normalem Tempo,

- Klasse 3: bereits bei reduziertem Fußgängertempo auf einem ebenen Weg,

- Klasse 4: bei geringster Belastung, wie z. B. Zähneputzen, sich Waschen oder sich Anziehen.

Vor allem Klasse 4 wird regelmäßig missinterpretiert als „Dyspnoe in Ruhe“. Und in den medizinischen Dokumentationen ist ohne Benutzung von konkreten Fragen die wahrscheinlich am häufigsten angegebene Klasse „2-3“, was völlig nutzlos ist.

\section{6-min-Gehtest}

Der 6-min-Gehtest ist heute bei chronischer Dyspnoe die Untersuchung der Wahl, um eine körperliche Limitation zu quantifizieren [2]. Der Patient geht auf einer geraden Strecke während 6 min hin und her. Dabei darf er nicht angespornt, sondern nur jede Minute über die noch verbleibende Zeit informiert werden. Obwohl der Sollwert dieses Testes nur approximativ mittels einer Formel bestimmt werden kann, spricht eine Gehstrecke von $>450 \mathrm{~m}$ für eine nur leichte und eine solche $<150$ m für eine schwere körperliche Einschränkung. Am Ende des Gehtests wird der Grad der Erschöpfung mit der von 1 (sehr leicht) bis 10 (sehr schwer) reichenden Borg-Skala dokumentiert. Die alte, von 6 bis 20 reichende Skala sollte nicht mehr verwendet werden.

\section{Gesundheitsbezogene Lebensqualität}

Neben dem Schweregrad der Dyspnoe, der 6-min-Gehstrecke und der Lungenfunktion ist die Analyse der Lebensqualität für eine umfassende Beurteilung eines Patienten unabdingbar. Dabei unterscheidet man zwischen sog. generischen, die allgemeine Lebenszufriedenheit messenden und die für eine bestimmte Krankheit spezifischen, sog. gesundheitsbezogenen Fragebögen. Die generische Lebensqualität wird z. B. mit dem SF-36-Fragebogen, dem EURO-QOL-Instrument und dem Nottingham Health Profile (NHP) bestimmt und hauptsächlich als Parameter für klinische Studien verwendet.
Eine zusätzliche Blutgasanalyse ist unabdingbar zur Erfassung des $\mathrm{p}_{\mathrm{a}} \mathrm{CO}_{2}$, der alveoloarteriellen $\mathrm{O}_{2}$ Partialdruckdifferenz, des pH (und somit des Basenexzesses)

Der 6-min-Gehtest dient dazu, eine körperliche Limitation zu quantifizieren 


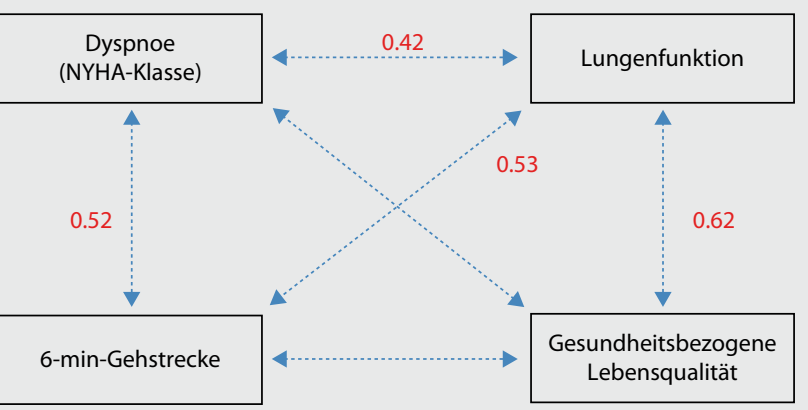

Abb. $1<$ Vier unabdingbare Parameter für eine umfassende Beurteilung des Patienten. Die Korrelation zwischen den 4 ist nur locker $(r=0,42-0,62)$. Einige aus der Literatur bekannte Werte sind in Rot angegeben
Die gesundheitsbezogene Lebensqualität sollte eigentlich bei jedem Patienten erfasst werden.

Für die COPD ist der „St. George's Respiratory Questionnaire" der bekannteste

Beim Asthma ist der „Juniper Asthma Quality of Life Questionnaire" der am häufigsten eingesetzte

Der CAMPHOR sollte in der alltäglichen Praxis routinemäßig verwendet werden

Das „einfache“ Schnarchen macht die Schnarcher selbst nicht krank
Ganz anders ist heute die Bedeutung der gesundheitsbezogenen Lebensqualität [3]. Sie sollte eigentlich bei jedem Patienten eingesetzt werden, ist doch die Korrelation zwischen Schweregrad des Dyspnoe, 6-min-Gehstrecke, Lungenfunktion und der Lebensqualität nur eine lockere (• Abb. 1). So kann ein Patient mit COPD und einer Erstsekundenkapazität von lediglich 30\% noch recht zufrieden sein und subjektiv nur wenig Atemnot verspüren. Anderseits ist unter Umständen ein Patient mit Lungenfibrose, einer Vitalkapazität von 70\% und einer 6-min-Gehstrecke von $500 \mathrm{~m}$ infolge seiner Tachypnoe und des chronischen quälenden Hustens massivst in seiner Lebensqualität eingeschränkt. Gut bekannt ist auch die Problematik des sog. „near-fatal“ Asthmaanfalles. Diese Patienten weisen oft eine verminderte Empfindung der Atemnot auf, was dazu führt, dass sie die Verschlechterung der Lungenfunktion erst quasi 5 vor 12 verspüren. Dies ist mitunter ein Grund, weshalb jeder Asthmapatient täglich seinen Peak Flow messen sollte.

Die gesundheitsbezogene Lebensqualität stellt heute einen der wichtigsten Endpunkte für klinische Studien dar. Sie ist auch für ökonomische Betrachtungen essenziell, werden doch aus den daraus ermittelten qualitätsassoziierte Lebensjahre ermittelt. Und letztlich ist sie wesentlich für die Beurteilung des Verlaufes einer Krankheit und somit für die alltägliche Praxis [4]. Für die COPD ist der „St. George's Respiratory Questionnaire“ (SGRQ) wohl der bekannteste [5]. Er beinhaltet 76 krankheitsbezogene Fragen, unterteilt in 3 Gruppen (Symptome, allgemeine Aktivität und Auswirkung auf das eigene Dasein). Weitere oft verwendete Instrumente sind der „Chronic Respiratory Disease Questionnaire“ (CRDQ) und der neue „Living with COPD Questionnaire“ (LCOPD). Beim Asthma ist wohl der „Juniper Asthma Quality of Life Questionnaire“ (AQLQ-Juniper) der am häufigsten eingesetzte. Neben dem „Asthma Control Questionnaire“ (ACQ) und dem „Pediatric Asthma Quality of Life Questionnaire“ (PAQLQ; [6]) wurde auch hier kürzlich mittels neuester ausgeklügelter Methodik analog dem LCOPD die „Asthma Life Impact Scale“ (ALIS) erstellt und validiert.

Dieselbe Arbeitsgruppe hat auch für die pulmonale Hypertonie den gesundheitsbezogenen Lebensqualität-Fragebogen „Cambridge Pulmonary Hypertension Outcome Review“ (CAMPHOR) entwickelt und validiert [7]. Das Instrument ist bereits in viele Sprachen übersetzt worden und spielt bei dieser seltenen und nur mit sehr teuren Therapien behandelbaren Krankheit eine extrem wichtige Rolle, einerseits für ökonomische Kosten-Nutzen-Aspekte und anderseits für die Ausarbeitung großer randomisierter, kontrollierter Studien, da hier der Mangel an zuverlässigen Studienendpunkten nach wie vor ein riesiges Problem darstellt. Der CAMPHOR sollte heutzutage auch in der alltäglichen Praxis routinemäßig verwendet werden, da bei dieser auch unter Therapie oft progredienten Krankheit ein sog. „zielorientierter Approach“ angewendet wird. Dazu braucht es, wie oben erwähnt, einigermaßen zuverlässige prognostische Parameter. Es ist eine noch wenig bekannte Tatsache, dass im Vergleich zu den anderen 3 wichtigen Endpunkten (NYHA-Klasse, 6-min-Gehstrecke, Herzminutenvolumen) der CAMPHOR die beste Sensitivität bezüglich einer für den Patienten spürbaren Verschlechterung („minimal clinically important difference“) hat.

\section{Schnarchen, Apnoe und Hypersomnie}

Schnarchen ist häufig (etwa 50\% der über 50-jährigen Männer). Das „einfache“ Schnarchen, das Lautstärken bis zu $80 \mathrm{~dB}$ erreichen kann, ist eine akustische Störung für die Umgebung, macht aber die Schnarcher selbst nicht krank. Es kommt durch ein Flattern der im Schlaf erschlafften oberen Atemwege zustande. Kommt es durch die intermittierende Widerstandserhöhung zu Aufwachreaktionen (,arou- 
sals") und damit zu einer Fragmentierung des Schlafmusters, treten Symptome auf. Diese bestehen aus vermehrter Einschlafneigung tagsüber und neuropsychologischen Störungen (Vergesslichkeit, Depression etc.). Solche Störungen des Schlafs treten insbesondere beim eigentlichen obstruktiven Schlafapnoesyndrom auf. Dabei kommt es intermittierend, bis 1- bis 2-mal pro Minute zu einem vollständigen Verschluss der oberen Atemwege trotz fortgesetzter Atembewegungen des Zwerchfells (paradoxes Atemmuster von Abdomen und Thorax). Sistiert die Atmung für 10 und mehr Sekunden, spricht man von einer Apnoe (Fremdanamnese, Tonbandaufzeichnung). Das Schlafapnoesyndrom ist häufiger bei Männern und wird durch Übergewicht begünstigt.

Zur Quantifizierung des Symptoms der vermehrten Einschlafneigung (Hypersommnie) dient der Epworth-Sleepiness-Scale [8]. Dabei wird erfragt, wie leicht es dem Patienten fällt, in 8 definierten alltäglichen Situationen einzuschlafen. Gemeint ist nicht nur das Gefühl, müde zu sein, sondern, auch wirklich einzuschlafen. Die Fragen beziehen sich auf das übliche tägliche Leben der vergangenen Wochen. Die Wahrscheinlichkeit einzuschlafen wird für jede Frage bzw. Situation mit 0 bis 3 Punkten bewertet:

- 0 = würde nie einschlafen,

- 1= würde kaum einschlafen,

- 2 = würde möglicherweise einschlafen,

- 3 = würde mit großer Wahrscheinlichkeit einschlafen.

Die Fragen lauten: Wie leicht fällt es Ihnen einzuschlafen bei folgenden Situationen:

I. Sitzen und Lesen,

II. Fernsehen,

III. Sitzen an einem öffentlichen Ort (z. B. Theater, Sitzung, Vortrag),

IV. als Mitfahrer im Auto während einer Stunde ohne Halt,

V. Abliegen, um auszuruhen am Nachmittag, wenn es die Umstände erlauben,

VI. Sitzen und mit jemandem sprechen,

VII. ruhig sitzen nach Mittagessen ohne Alkohol,

VIII. im Auto beim Stopp an einer Verkehrsampel während einiger Minuten.

Der Score reicht somit von 0 bis 24. Er dient v. a. der Einschätzung des Schweregrades und des Verlaufes eines Schlafapnoesyndroms. Während Gesunde einen Score von 10 und weniger aufweisen, liegen die Werte bei einem Schlafapnoesyndrom meistens höher. Die häufigsten Ursachen für eine vermehrte Einschlafneigung sind aber v. a. Schlafmangel, schlechte Schlafhygiene und medizinische Probleme wie gastroösophagealer Reflux oder Herzinsuffizienz.

Die Narkolepsie ist eine seltene Störung einhergehend mit

- kurzen, oft wiederholten Schlafattacken, die nicht nur bei den oben beschriebenen schlaf-

fördernden Situationen, sondern auch bei normaler körperlicher Aktivität auftreten können,

- Kataplexie (abrupter, reversibler, einige Sekunden bis 30 min dauernder Verlust des

Muskeltonus, häufig ausgelöst durch Emotionen),

- Schlaflähmung (Gefühl beim Einschlafen oder Aufwachen, sich nicht mehr bewegen zu können) und oft damit einhergehend

- hypnagoge Halluzinationen (Träume beim Einschlafen bzw. Aufwachen).

\section{Andere Symptome bei Lungenkrankheiten}

Der Husten ist ein komplexer physiologischer Abwehrmechanismus. Häufige Ursachen eines akuten Hustens sind eine Aspiration (Frage nach Verschlucken, Dysphagie) und eine akute Bronchitis (Frage nach grippalen Symptomen). Die wichtigste Ursache eines chronischen Hustens ( 3 Wochen) ist die chronische Bronchitis (Definition gemäß WHO: täglicher Husten während mindestens 3 aufeinanderfolgenden Monaten während 2 Jahren). In weit über $90 \%$ sind dabei Raucher mit oder ohne COPD betroffen. Andere Differenzialdiagnosen sind eine postvirale bronchiale Hyperreagibilität, ein „Post-Nasal-Drip-Syndrom“ im Rahmen einer chronischen Sinusitis (Gesichts- oder Kopfschmerzen, „Eiterstraße“ an der Rachenhinterwand), ein nicht diagnostiziertes Asthma bronchiale (nächtlicher Husten, Zunahme des Hustens in Kälte, während oder nach Belastung,während Pollensaison), ein gastroösophagealer Reflux, ein Lungenkarzinom (Raucheranamnese), Medikamente (v. a. ACE-
Zur Quantifizierung des Symptoms der vermehrten Einschlafneigung (Hypersomnie) dient der EpworthSleepiness-Scale

Die wichtigste Ursache eines chronischen Hustens ist die chronische Bronchitis 
Bei der Hämoptoe unterscheidet man 2 Schweregrade: die Minorund die Major-Hämoptoe

Thoraxschmerzen sind meistens ein Alarmsymptom

Beim Stridor handelt es sich um ein mit bloßem Ohr hörbares, hochfrequentes Geräusch in Abhängigkeit zum Atemzyklus
Hemmer) und die Lungenfibrose. Auch eine kardial bedingte Lungenstauung kann mit Husten, der im Liegen oder bei Anstrengung zunimmt, als einziges Symptom einhergehen.

Bei der Hämoptoe unterscheidet man 2 Schweregrade (wie so oft in der Medizin unter Verwendung von Anglizismen):

- die „Minor-Hämoptoe“: nur blutig tingiertes Sputum,

— die „Major-Hämoptoe“: reines Blut, Quantität unterschiedlich: wenige Milliliter bis mehrere hundert Milliliter.

Bei der Ersteren sind häufige Ursachen eine hämorrhagische Bronchitis (Leck eines kleinen Blutgefäßes bei akuter oder chronischer Bronchitis), ein Lungenkarzinom, eine Lungenembolie sowie allenfalls eine Lungenstauung bei Herzinsuffizienz. Häufige Ursachen für eine „Major-Hämoptoe“ sind die Ruptur einer Bronchialarterie bei Bronchiektasen, ein Lungenkarzinom mit Arrosion einer größeren Pulmonalarterie, Tuberkulose, Aspergillom, Karzinoid sowie arteriovenöse Malformationen.

Besteht ein produktiver Husten, soll bei der Untersuchung Sputum gewonnen und inspiziert werden. Man bittet den Patienten, mit starken Hustenstößen das Sekret von tief unten heraufzuhusten und in ein bereitstehendes Gefäß zu spucken. Wird zur diagnostischen Zwecken bei Verdacht auf Pneumozystis-Pneumonie bei HIV-Patienten Sputum benötigt und vom Patient aber keines produziert, kann mittels Inhalation von 3\%iger Kochsalzlösung in den meisten Fällen Sputum provoziert werden.

Bereits der makroskopische Aspekt des Sputums kann gewisse differenzialdiagnostische Hinweise geben, je nachdem ob die Sputumbeschaffenheit weißlich-mukös (Asthma bronchiale, Lungenfibrose, bronchioloalveoläres Karzinom), gelblich-eitrig (akute Bronchitis, Pneumonie, Bronchiektasen, Lungenabszess, Tuberkulose), grünlich-eitrig (Pseudomonas-Infekt), eitrig-stinkend (Anaerobier-Abszess), schaumig und evtl. blutig tingiert (kardiales Lungenödem) oder eben blutig (s. oben) ist. Thoraxschmerzen sind meistens ein Alarmsymptom. Atemabhängige Schmerzen sprechen, abgesehen von der akuten Perikarditis, zumeist für eine pulmonale (oder gastrointestinale) Affektion. Differenzialdiagnostisch kommen v. a. eine Pleuropneumonie, eine akute (virale) Pleuritis, ein Pneumothorax und eine Lungenembolie infrage. Besteht eine lokale, starke Druckdolenz ist das diagnostisch für eine (spontane) Rippenfraktur. In diesem Zusammenhang ist zu betonen, dass dies eine klinische Diagnose darstellt. Ein Röntgenbild ist für die Diagnose unbrauchbar und dient lediglich dem Ausschluss einer anderen Ursache, v. a. eines Pneumothorax.

Dumpfe, nicht genau lokalisierbare Thoraxschmerzen mit Druckgefühl retrosternal und ggf. Ausstrahlung in Kiefer und linken Arm, evtl. verstärkt bei Anstrengung sind klassisch für eine Angina pectoris. Atemabhängige Schmerzen schließen die Diagnose praktisch aus. Pathognomonisch für eine akute Aortendissektion ist die Trias perakuter, vernichtender und interskapulär ausstrahlender Thoraxschmerz, der von den Schultern in die unteren Extremitäten wandern kann, und eine Blutdruckdifferenz links-rechts oder oben-unten. Beim Stridor handelt es sich um ein mit bloßem Ohr hörbares, hochfrequentes Geräusch in Abhängigkeit zum Atemzyklus. Ein vorwiegend inspiratorischer Stridor tritt bei variablen extrathorakalen Atemwegsstenosen (Larynx), ein inund exspiratorischer Stridor bei fixierten intra- oder extrathorakalen Stenosen (Struma, zentrales Lungenkarzinom) auf.

\section{Physikalische Untersuchung der Lungen}

\section{Inspektion}

Die Thoraxform entspricht bei vielen Gesunden dem athletischen oder asthenischen Typ: Symmetrie und ein epigastrischer Winkel von $90^{\circ}$ sind die Hauptkennzeichen. Abweichungen von dieser Form sind konstitutionelle Spielarten des Normalen. Leichte Thoraxdeformitäten sind häufig und haben keine Auswirkung auf die Lungenfunktion. Eine schwere Kyphoskoliose hingegen verursacht eine restriktive Ventilationsstörung und kann zu einer progredienten respiratorischen Insuffizienz führen. Die Trichterbrust (Pectus excavatum) ist gekennzeichnet durch eine Eindellung des kaudalen Anteils des Sternums. In schwereren Fällen sind das ganze Sternum und auch der knorpelige Anteil der Rippen abgesenkt. Selten ist dann einmal eine chirurgische Korrektur notwendig. Unter einer Hühnerbrust (Pectus carinatum) versteht man das schiffskielähnliche Hervortreten des Brustbeins bei nach vorne gelagerten Rippenansätzen, was aber ohne krankheitsrelevante Bedeutung ist. 


\begin{abstract}
Abb. $2 \triangleright$ Die maximale laryngeale Distanz (Abstand zwischen Jugulum und der Inzisur am Oberrand des Schildknorpels) beträgt normalerweise bei rekliniertem Kopf mehr als $4 \mathrm{~cm}$
\end{abstract}

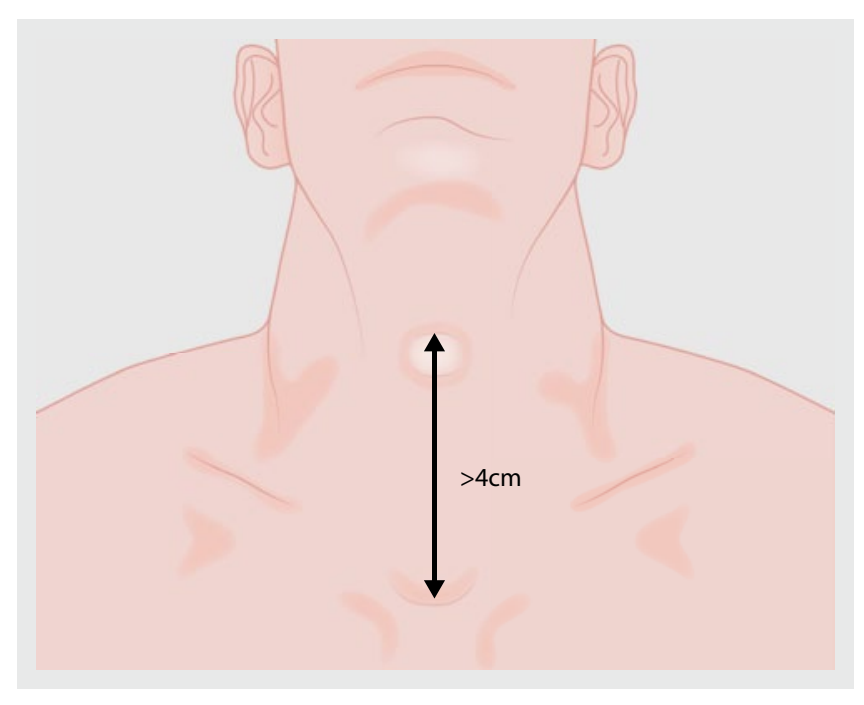

Deformationen des Thorax mit Einziehen eines Hemithorax können sich bei einer Pleuraschwarte, nach Pneumektomie oder nach einer Thorakoplastik entwickeln. Ist der sagittale Thoraxdurchmesser verlängert und somit der Thoraxquerschnitt rund und nicht mehr oval, so spricht man etwas despektierlich von einem Fassthorax. Dies ist der Fall bei ausgeprägtem Lungenemphysem, bei dem es aufgrund des Elastizitätsverlustes der Lunge zur Überblähung mit konsekutiven Veränderungen der Thoraxform kommt. Der Brustkorb befindet sich in einer fast fixierten Einatmungsstellung. Das Brustbein steht höher, die Rippen verlaufen horizontal, und die Interkostalräume sind verbreitert. Zudem wird die obere Thoraxapertur nach kranial verlagert, sodass der Abstand zwischen Jugulum und der Inzisur am Oberrand des Schildknorpels (• Abb. 2), die sog. maximale laryngeale Distanz bei rekliniertem Kopf („maximal laryngeal height"), auf $4 \mathrm{~cm}$ oder weniger abnimmt [9].

Die Beurteilung der Atmung beinhaltet die Messung der Atemfrequenz, des Atemrhythmus, der Atemsymmetrie und -synchronie. Im Liegen werden beim Gesunden während der ruhigen Normalatmung rund zwei Drittel des Atemzugvolumens durch den Zwerchfellanteil der Atmung (abdominales Kompartiment) verschoben. Im Stehen verhält es sich umgekehrt, indem die Thoraxexkursion den größeren Anteil beiträgt (thorakale Atmung). Der unterschiedliche Beitrag von Thorax und Abdomen ist eine Folge der je nach Körperlage unterschiedlichen Zwerchfellposition in der Atemruhelage. Es gibt keinen Unterschied im Atemtyp thorakal vs. abdominal zwischen Männern und Frauen. Wichtig ist der Hinweis, dass die Beurteilung der Atmung und v. a. ihrer Symmetrie und Synchronie durch die Palpation der entsprechenden Bereiche unterstützt werden sollte (vgl. • Abb.4). Mittels „palpationsunterstützter Inspektion“ sind diskrete Veränderungen viel besser feststellbar.

Die Atemfrequenz ist das pulmonale Vitalzeichen. Die normale Atemfrequenz des Erwachsenen beträgt 8 bis 20 pro Minute. Sie kann natürlich nicht im eigentlichen Sinne inspiziert, sondern muss „unauffällig“" während der Anamnese bestimmt werden. Das normale Verhältnis der zeitlichen Dauer von Inspiration und Exspiration beträgt 2:3. Eine bronchiale Obstruktion (Asthmaanfall, exazerbierte COPD) verlängert das Exspirium.

Eine flache und beschleunigte Atmung findet man bei einer Lungenfibrose oder Pleuritis. Die Tachypnoe ist eines der wichtigsten klinischen Zeichen und kommt neben Lungen- und Herzkrankheiten auch bei Lungenembolie oder psychogen (Panikattacke) vor. Sie ist definiert durch eine Atemfrequenz $>20 / \mathrm{min}$. Eine erhöhte Atemfrequenz stellt grundsätzlich einen Alarmbefund dar und ist z. B. bei einer Pneumonie eine Indikation für eine sofortige Krankenhauseinweisung. Eine Bradypnoe (Atemfrequenz $<8 / \mathrm{min}$ ) wird bei Opiatintoxikation beobachtet. Als Kussmaul-Atmung wird eine langsame und in Folge vergrößerter Atemzugvolumina vertiefte Atmung bezeichnet, die bei metabolischer Azidose auftritt. Eine eingeschränkte Thoraxexkursion $<3 \mathrm{~cm}$ gilt als eines der diagnostischen Kriterien bei M. Bechterew.

Die Atemperiodik der Cheyne-Stokes-Atmung ist gekennzeichnet durch eine zyklische $\mathrm{Zu}$ und Abnahme der Atemtiefe und Apnoen (- Abb. 3). Die häufigste Ursache ist eine Verlänge-
Die Beurteilung der Atmung beinhaltet die Messung der Atemfrequenz, des Atemrhythmus, der Atemsymmetrie und-synchronie

Die Tachypnoe (Atemfrequenz $>20 / \mathrm{min}$.) ist eines der wichtigsten klinischen Zeichen und stellt stets einen Alarmbefund dar. 

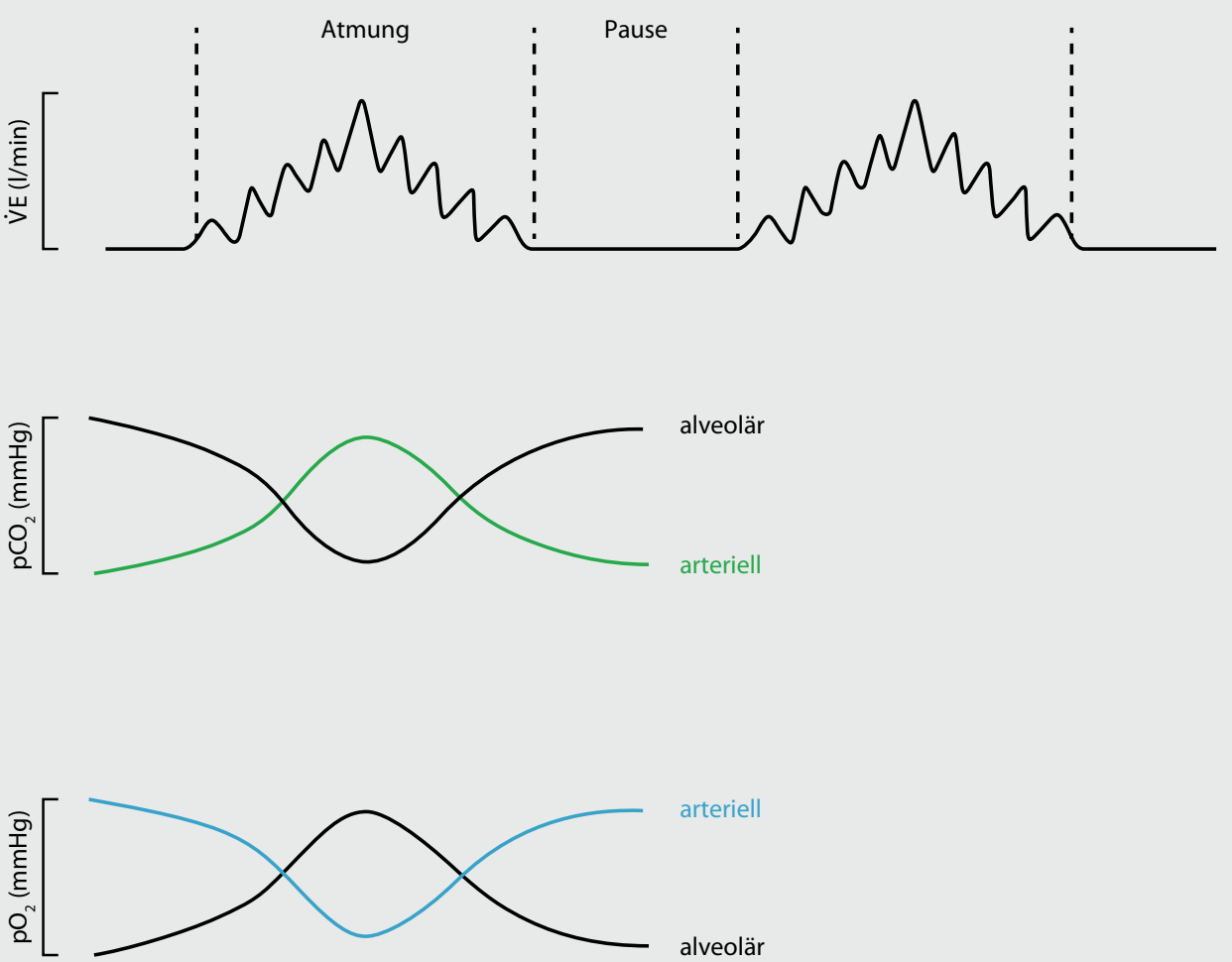

Abb. $3 \Delta$ Bei der Cheyne-Stokes-Atmung kommt es in der Ventilationsphase naturgemäß zu einem Abfall des alveolären $\mathrm{p}_{\mathrm{a}} \mathrm{CO}_{2}$. Die gleichzeitige Erhöhung des alveolären $\mathrm{p}_{\mathrm{a}} \mathrm{O}_{2}$ wirkt sich aber erst verspätet auf das arterielle $\mathrm{p}_{\mathrm{a}} \mathrm{O}_{2}$ aus, dessen Anstieg nach der Ventilationsphase dann eine Atempause erzeugt

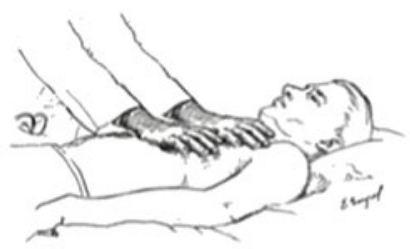

Thorakale Atmung (Atemmuskulatur)

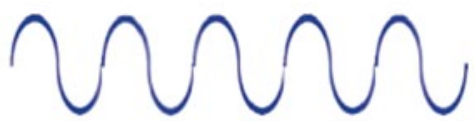

Adominale Atmung (Zwerchfellatmung)

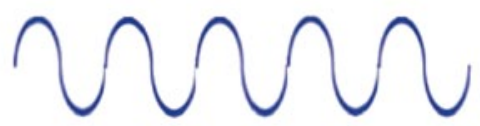

Paradoxe Atmung
Thorax

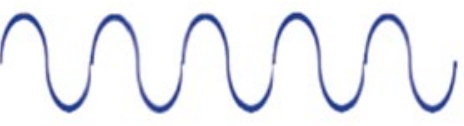

Abdomen (Zwerchfell)

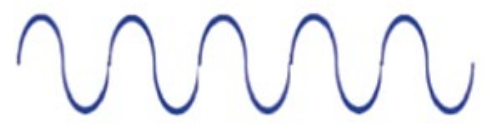


flächlichen Atemzügen und apnoischen Pausen. Dieses Phänomen wird bei Intoxikationen, schwerer zerebraler Schädigung und in der Agonie beobachtet. Seufzer sind unwillkürliche, mehrmals pro Stunde auftretende, vertiefte Atemzüge. Sie sind physiologisch und dienen zur Wiedereröffnung von kleinen kollabierten Lungenabschnitten (Mikroatelektasen).

Durch eine Überblähung der Lungen (Elastizitätsverlust bei Emphysem, erhöhter exspiratorischer Widerstand) kommt es zur Abflachung des Zwerchfells. Dieses ist dadurch als Inspirationsmuskel in einer ungünstigen Ausgangsposition (Laplace-Gesetz). In dieser Situation wird die auxiliäre Atemmuskulatur rekrutiert. Man kann eine sicht- und tastbare Aktivierung des M. sternocleidomastoideus, der Mm. scaleni und des M. trapezius feststellen. Zur aktiven Exspiration wird zudem die Bauchmuskulatur (Bauchpresse) gebraucht. Bei schwerer Lungenüberblähung (Lungenemphysem) sind die Zwerchfelle völlig flach oder sogar leicht nach kaudal gekrümmt. Ihre Kontraktion kann zu einer inspiratorisch sichtbaren lateralen Einziehung der unteren Thoraxapertur führen („Hoover-Zeichen“). Der stark dyspnoische Patient mit obstruktiver Ventilationsstörung stützt sich häufig in sitzender Position auf den Schultergürtel ab, was die Wirksamkeit der Atemhilfsmuskulatur begünstigt. Zudem atmet er oft durch die halb geschlossenen Lippen aus (Lippenbremse), um den häufigen exspiratorischen Bronchialkollaps zu vermindern. Fällt das Zwerchfell (Innervation: C3-4) als wichtigster Inspirationsmuskel im seltenen Fall einer bilateralen Parese aus, z. B. bei einer Infektion durch das Frühsommermeningoenzephalitis-Virus, ist die Atmung nur noch im Sitzen möglich. Dies wird als Orthopnoe bezeichnet und ist ansonsten ein sehr spezifisches Zeichen für eine Linksherzinsuffizienz.

Das Gegenteil ist die sog. Platypnoe (oder Orthodeoxia-Platypnoe-Syndrom), was bedeutet, dass es im Sitzen zu einer Sauerstoffdesaturation und somit zu Atemnot kommt. Dieses Phänomen ist zumeist Folge eines Rechts-links-Shunts, der im Sitzen verstärkt wird. Ursachen sind meist basal liegende AV-Malformationen (M. Osler, hepatopulmonales Syndrom) und selten ein offenes Foramen ovale oder ein Vorhofseptumdefekt.

Eine asymmetrische Atmung mit einer einseitigen Einschränkung der Thoraxbewegungen wird bei anatomisch bedingter Fixation (Pleuraschwarte), Pleuraerguss, einseitiger Zwerchfelllähmung (idiopathisch, nach Herzoperationen etc.) nach Lungenresektion sowie bei reflektorischer Schmerzhemmung (Pleuritis, Lungenembolie mit Pleurareizung, Rippenfraktur) beobachtet.

Eine nichtsynchrone Bewegung von Thorax und Abdomen wird als paradoxe Atmung bezeichnet (- Abb. 4). Bei der physiologischen inspiratorischen Auswärtsbewegung des Thorax kommt es in diesem Fall zu einer passiven Einwärtsbewegung des Abdomens, zumeist verursacht durch die Ermüdung der Zwerchfellmuskulatur (länger anhaltende Erhöhung der Atemarbeit, verminderte Zwerchfelldurchblutung im Schock) und insbesondere bei gleichzeitiger Überblähung des Zwerchfells (exazerbierte COPD, schweres Asthma). Dies ist ein untrügliches Zeichen einer nahenden respiratorischen Erschöpfung bzw. der Notwendigkeit einer maschinellen Beatmung. Neben der Atemfrequenz und dem subjektiven Befinden des Patienten ist eine paradoxe Atmung bei der Indikationsstellung einer Beatmung weitaus wichtiger als andere Parameter, wie z. B. die Blutgaswerte.

Trommelschlegelfinger und Uhrglasnägel („clubbing“) werden praktisch ausschließlich bei idiopathischer Lungenfibrose und zystischer Fibrose, Bronchiektasen, Lungentumoren und praktisch nie bei anderen Lungenkrankheiten, wie z. B. COPD, gefunden. Im Rahmen von Lungentumoren kann es zu einer hypertrophen Osteoarthropathie (Pierre-Marie-Bamberger) mit schmerzhaften Gelenkschwellungen und Knochenneubildung an den langen Röhrenknochen kommen. Häufigste Ursache ist neben dem Lungenkarzinom (paraneoplastisch) die zystische Fibrose. Die Ätiologie dieser Veränderungen ist unbekannt. Während früher das Bonmot galt, Trommelschlegelfinger sind vorhanden, wenn der Erfahrenste es so sagt, gibt es heute dafür klare klinische Kriterien, die zumindest einen internationalen Konsens darstellen (• Abb. 5).

\section{Palpation}

Wie oben erwähnt, spielt die Palpation bei der Inspektion von Atemrhythmus, -symmetrie, -synchronie und Atemhilfsmuskulatur eine wichtige unterstützende Rolle. 


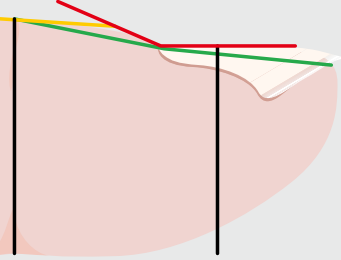

a

b

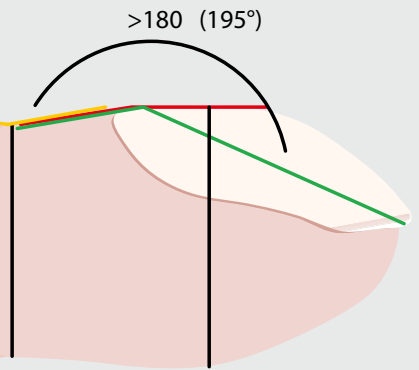

$\mathrm{IPD}<\mathrm{DPD}$

Abb. $5 \Delta$ a-d Kriterien für das Vorliegen von Trommelschlegelfingern („clubbing“). b Der distale Fingerdurchmesser („distal phalangeal depth“, DPD) ist größer als der interphalangeale Durchmesser im Bereiche des Gelenks (,interphalangeal depth“, IPD). Der hyponychiale Winkel (grüne Linie) beträgt $>180^{\circ}$ („,offiziell“, aber didaktisch ungeschickt $>195^{\circ}$ ). d Beim Schamroth-Zeichen gibt es beim Zusammenhalten beider Daumenendglieder keinen Spalt mehr, sondern die Fingernägel gehen schnabelartig auseinander

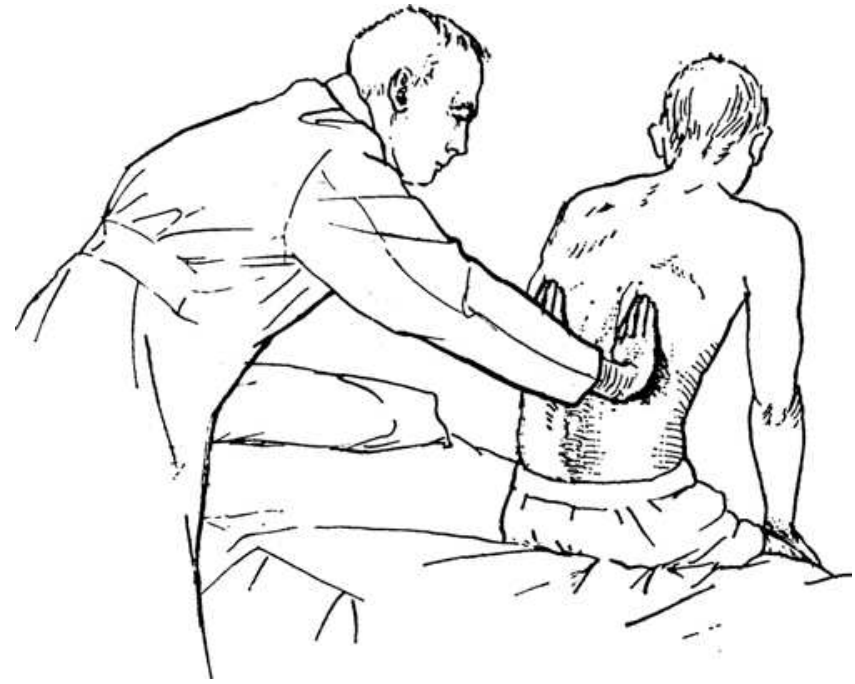

Abb. $6<$ Untersuchung des Stimmfremitus. (Aus [20]) 
Abb. 7 Der Zeigefinger der einen Hand wird als Plessimeter (gr. $\pi \lambda \varepsilon \dot{\sigma} \sigma \varepsilon \varepsilon$ = klopfen) auf den Thorax gelegt und dann mit zusammengelegtem Zeige- und Mittelfinger beklopft. (Aus [20])

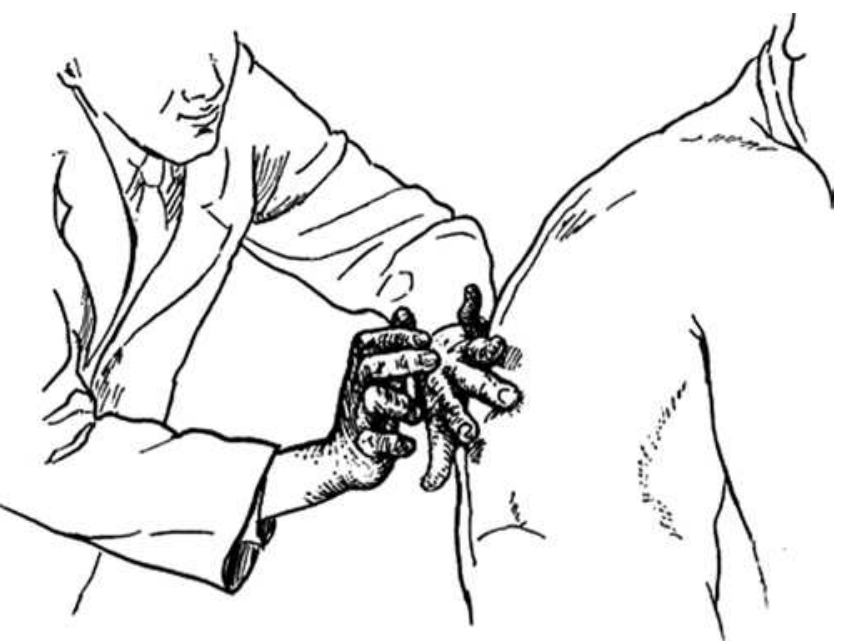

Pneumothorax). Ein pathologischer Stimmfremitus kann natürlich nur bei einseitigen Lungenerkrankungen bestimmt werden.

\section{Perkussion}

Die Perkussion (• Abb. 7) dient einerseits der Feststellung der unteren Lungengrenzen (abgrenzende Perkussion), und andererseits der vergleichenden Prüfung korrespondierender Abschnitte der rechten und linken Lunge (vergleichende Perkussion).

Bei der abgrenzenden Perkussion beginnt man mit dem „Klopfen“ über dem lufthaltigen Lungengewebe dorsal bzw. lateral und bewegt sich dann nach kaudal. Die unteren Lungengrenzen befinden sich direkt paravertebral auf Höhe der 10. Rippe, in der Skapularlinie auf Höhe der 9. Rippe und in der mittleren Axillarlinie auf Höhe der 8. Rippe. Je nach Abweichung von diesen Grenzen kann eine Restriktion oder eine Überblähung vermutet werden. Oft ist dann auch die respiratorische Verschieblichkeit des Zwerchfells aufgrund der Perkussion bei maximaler In- und Exspiration auf $<4 \mathrm{~cm}$ vermindert.

Bei der vergleichenden Perkussion sucht man, ob die Schallqualitäten der beiden Lungen identisch oder einseitig verändert (hypersonor oder gedämpft) sind. Eine Dämpfung ist dann vorhanden, wenn sich kein lufthaltiges Gewebe mehr unter der Thoraxwand befindet (Pleuraerguss, Pleuraschwarte, Konsolidation von Lungenparenchym, Zwerchfellparese). Ein hypersonorer Klopfschall findet sich bei vermehrtem intrathorakalem Luftgehalt (Pneumothorax).

\section{Auskultation}

Der Patient sollte, wenn möglich, sitzend oder stehend untersucht werden. Man lässt ihn durch den geöffneten Mund atmen. Alle Lungenabschnitte vorne und hinten werden während mindestens einer ganzen Atemphase auskultiert.

Das Atemgeräusch entsteht durch turbulenten Luftfluss in den zentralen Atemwegen ( $\bullet$ Abb. 8). Dieser „Tongenerator“ ist direkt über der Trachea als fauchendes Geräusch hörbar. Das normale Atemgeräusch ist nichts anderes als das durch die lufthaltige Lunge wegfiltrierte Geräusch des zentralen „Tongenerators“. Ein abnormes Atemgeräusch kann nur bei einseitigen bzw. lokalisierten Veränderungen festgestellt werden. Entsprechend den beim Stimmfremitus erläuterten physikalischen Mechanismen kann das Atemgeräusch verschärft oder abgeschwächt sein. Hört man in dieser Situation keine Nebengeräusche („silent chest“) lässt man den Patienten ein Tiffenau-Manöver durchführen, wodurch erstens das Geräusch hörbar und zweitens die forcierte exspiratorische Zeit messbar ist. Beträgt Letztere mehr als 9 s, liegt mit großer Wahrscheinlichkeit eine obstruktive Ventilationsstörung vor.

Die pulmonalen Nebengeräusche werden aufgrund ihrer akustischen Eigenschaften in Giemen (oder Pfeifen), Brummen, feine und grobe Rasselgeräusche (Rasseln) eingeteilt. Giemen (Pfeifen) und Brummen entstehen durch Oszillation der pathologisch veränderten und/oder verengten Bronchi-

Die abgrenzende Perkussion dient der Feststellung der unteren Lungengrenzen

Bei der vergleichenden Perkussion prüft man, ob die Schallqualitäten korrespondierender Abschnitte der beiden Lungen verändert sind

Ein abnormes Atemgeräusch kann nur bei einseitigen bzw. lokalisierten Veränderungen festgestellt werden

Die pulmonalen Nebengeräusche werden in Giemen, Brummen, feine und grobe Rasselgeräusche eingeteilt 


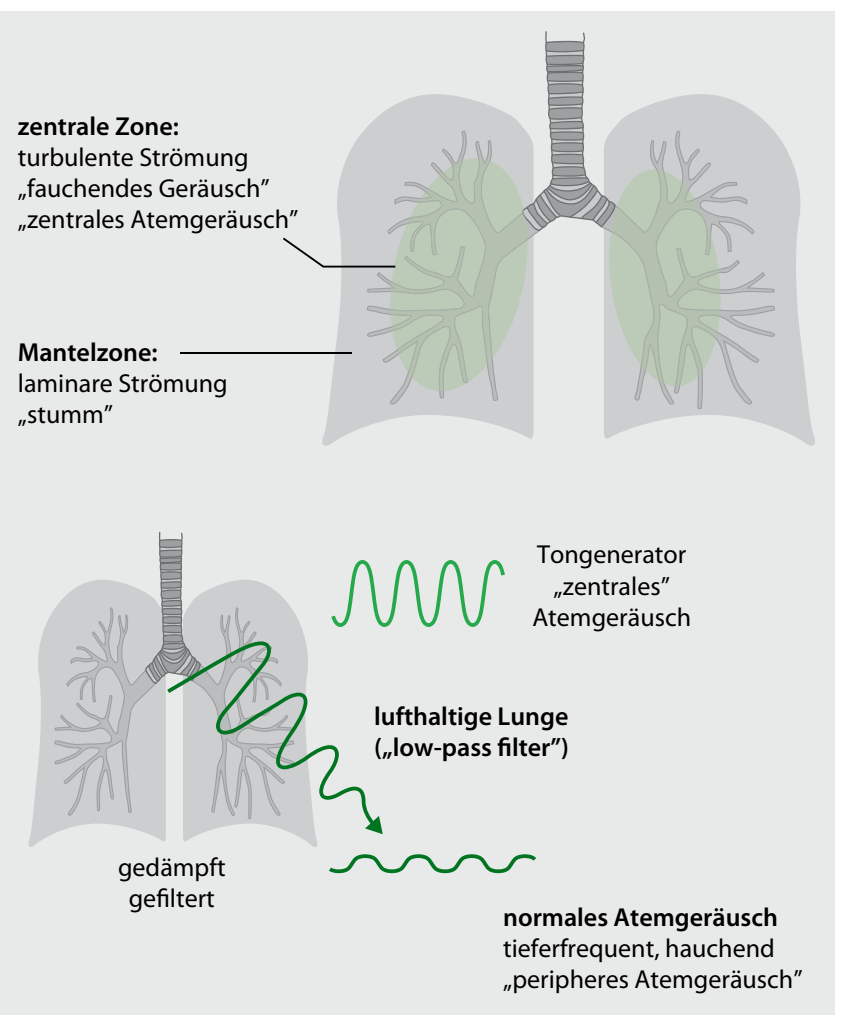

Abb. $8<$ Das normale Atemgeräusch entsteht durch Abfilterung der hohen Frequenzen des durch Turbulenzen in den größeren Atemwegen erzeugten fauchenden „zentralen“ Atemgeräuschs

en („Materialschwingungen“ aufgrund des Bernoulli-Gesetzes während der Exspiration, d. h. bei obstruktiven Ventilationsstörungen). Rasselgeräusche hingegen sind intermittierende kurze Geräusche, die durch den v. a. in- aber auch exspiratorischen Luftstrom durch mehr oder weniger große sekrethaltige Bronchien zustande kommen (Bronchitis, Lungenödem, Pneumonie). Es muss betont werden, dass die Alveolen normalerweise keine Geräusche machen (vgl. Unwort „vesikuläres“ Atemgeräusch), da sich dort ja keine Luft bewegt. Beispielhaft dafür ist die Pneumozystis-Pneumonie, die lediglich ein Alveolen-füllender Prozess ist und deshalb mit dem Bild von bilateralen azinären Infiltraten im Thoraxröntgen und fehlenden Nebengeräuschen einhergeht.

Analog der Physik des Stimmfremitus können die Rasselgeräusche bei einer Pneumonie noch als „klingend“ umschrieben werden, was aber keine „offizielle“ Bezeichnung darstellt. Ohrnahe hochfrequente beidseitige Rasselgeräusche („Knisterrasseln“, „velcro-rales“) sind pathognomonisch für eine Lungenfibrose. Sind sie nur einseitig und dabei diskreter vorhanden, kann es sich auch um ein Pleurareiben oder eine Pneumonie handeln.

Es gibt interessante Untersuchungen über Art, Zeitpunkt und Ausdehnung der Rasselgeräusche [10]. Sind sie feinblasig, eher frühinspiratorisch und auch in den höheren Lungenabschnitten hörbar, spricht das für eine Lungenfibrose. Bei der COPD sind die Rasselgeräusche eher grobblasig, basal und über die ganze Inspirationszeit hörbar. Bei der Linksherzinsuffizienz sind sie häufiger fein- als grobblasig, basal und eher gegen Ende der Inspiration hörbar.

Ein inspiratorisches Quietschen („inspiratory squeak“) ist ein nicht seltenes Phänomen. Es wurde nur einmal in der Literatur 1977 durch Geddes [11] beschrieben, die Lungentransplantationsspezialisten kennen es aber sehr gut. Es ist ein pathognomonisches Zeichen für eine konstriktive Bronchiolitis obliterans.

Analog des Stimmfremitus kann bei der Auskultation die Bronchophonie durch Erzeugenlassen von Zischlauten, z. B. Flüstern von „66“, geprüft werden.

Abschließend muss mit Vehemenz betont werden, dass die oben genannten Begriffe auf einer eigentlich schon seit 1971 bestehenden und anlässlich eines Internationalen Lung Sound Symposiums bestätigten Nomenklatur beruhen [12, 13]. Alle anderen Unwörter wie „feucht“, „trocken“, „Vesikuläratmen“ oder „kontinuierlich“ und „diskontinuierlich“ etc. sind obsolet und sollten nicht mehr verwendet werden. 
Abb. 9 Probabilistischer diagnostischer Prozess mittels vereinfachtem Bayes-Theorem [20]

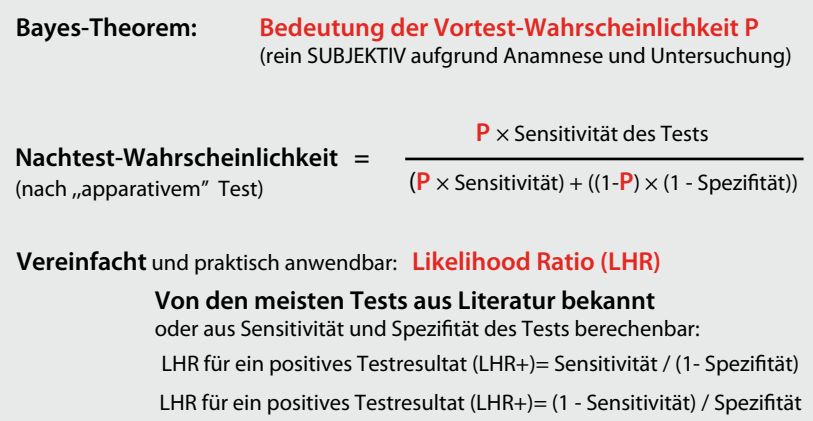

Nachtest-Chance $=$ Vortest-Chance $\times$ LHR

\section{Evidenzbasierte Anamnese und Untersuchung}

Anamnese und klinische Untersuchung werden gemeinhin als „lästige Notwendigkeiten“ betrachtet. Dabei ist es klar, dass sich auch heute in der Zeit der hoch technisierten Medizin mittels Anamnese und Untersuchung weit über $80 \%$ der Diagnosen stellen lassen [14, 15, 16, 17]. All die modernen Technologien sind zumeist nur dazu da, die subjektive Vermutungsdiagnose zu bestätigen.

Neben dieser Tatsache muss mit Nachdruck betont werden, dass die auf Anamnese und Untersuchung basierende, ganz persönliche subjektive Vortest-Wahrscheinlichkeit die Grundlage für die Bestimmung der Wahrscheinlichkeit für das Vorliegen einer Krankheit nach Erhalt des Testresultates (Laborwert, Röntgenbefund etc.) ist. Dies ist begründet auf dem Bayes-Theorem, das besagt, dass die (Nachtest-)Wahrscheinlichkeit einer Krankheit erstens durch die Qualität eines Tests (Sensitivität und Spezifität), aber auch durch die subjektiv eingeschätzte Vortest-Wahrscheinlichkeit bestimmt ist (• Abb. 9). Dies kann gut am Beispiel der Lungenembolie erklärt werden. Bei einer mittleren bis niedrigen Vortest-Wahrscheinlichkeit schließt ein negativer D-Dimer-Test eine Lungenembolie mit großer Wahrscheinlichkeit aus. Bei hoher subjektiver Vortest-Wahrscheinlichkeit ist eine Lungenembolie aber trotz negativem Test ziemlich wahrscheinlich, was heißt, dass man in dieser Situation gar kein D-Dimer bestimmen, sondern gleich eine Computerangiographie durchführen sollte. Anzumerken ist, dass die bekannten klinischen Scores zur Bestimmung der VortestWahrscheinlichkeit (Wells, Geneva) zwar didaktisch nützlich, aber keineswegs der rein subjektiven Einschätzung des erfahrenen Arztes überlegen sind.

Ein Problem dieses probabilistischen Vorgehens ist, dass oft behauptet wird, die Anwendung des Bayes-Theorems sei mathematisch kompliziert und deshalb in der Praxis nicht anwendbar. Dies ist aber keineswegs der Fall. Die etwas komplizierte Bayes-Formel kann nämlich einfach umgesetzt werden mittels der sog. Likelihood-Ratio (LHR) und unter Verwendung von Chancen statt Wahrscheinlichkeiten (• Abb. 9).

Eine bestimmte LHR+ besagt, dass die Nachtest-Chance für das Vorliegen einer Krankheit nach Durchführung eines apparativen Tests der subjektiven Vortest-Chance eines Arztes multipliziert mit der LHR+ entspricht. Die LHRs sind meistens aus der Literatur bekannt (• Tab. 1) oder können aus Sensitivität und Spezifität berechnet werden ( $\bullet$ Abb. 9). Nicht selten sind diese LHRs denjenigen von apparativen Tests, wie z. B. der Bestimmung des „brain natriuretic peptide“ (BNP), ebenbürtig (• Tab. 1). Mathematische Bedenken gibt es hierbei nicht, da es in praxi nur um grobe Einschätzungen geht, also z. B. einer hohen (4:1), mittleren (1:1) oder niedrigen (1:4) Vortest-Chance (entsprechend einer Vortest-Wahrscheinlichkeit von 80, 50 oder 20\%). Bei Tests mit hoher Sensitivität ist nur ein negatives Testresultat hilfreich, bei spezifischen Tests ein positives. Wie man aus - Tab. 1 entnehmen kann, beeinflussen Anamnese und Untersuchung die Wahrscheinlichkeit für das Vorliegen einer bestimmten Krankheit enorm. So erhöht die Kombination Nikotinabusus $>40$ "pack years", Alter $\geq 45$ Jahre und eine maximale laryngeale Distanz $\leq 4 \mathrm{~cm}$ die Chance für eine COPD um das 59-Fache! Andererseits verringert ein bekanntes Asthma bronchiale bei entsprechender Symptomatik die Chance für das Vorliegen einer Pneumonie um das 10-Fache.
Mittels Anamnese und Untersuchung lassen sich weit über $80 \%$ der Diagnosen stellen 


\begin{tabular}{|c|c|c|}
\hline & LHR+ & LHR- \\
\hline \multicolumn{3}{|l|}{ COPD } \\
\hline Giemen & 4,0 & 0,8 \\
\hline Forcierte exspiratorische Zeit $>9 \mathrm{~s}$ & 6,7 & NA \\
\hline Forcierte exspiratorische Zeit 6-9 s & 1,8 & 0,6 \\
\hline Peak Flow $\leq 150 \mathrm{ml}$ zur Differenzialdiagnose Herzinsuffizienz & 4,0 & 0,2 \\
\hline Nikotinabusus $>40$ "pack years" & 11,6 & 0,9 \\
\hline Maximale laryngeale Distanz $\leq 4 \mathrm{~cm}$ & 3,6 & 0,7 \\
\hline Alter $\leq 45$ Jahre & 2,1 & 0,7 \\
\hline Letzte 3 zusammen & 59 & 0,3 \\
\hline \multicolumn{3}{|l|}{ Pneumonie } \\
\hline Bronchophonie & 8,6 & 0,9 \\
\hline Rasselgeräusche & 2,7 & 0,8 \\
\hline Verschärftes Atemgeräusch & 3,5 & 0,9 \\
\hline Perkutorische Dämpfung & 4,3 & 0,8 \\
\hline Anamnese eines Asthma bronchiale & 0,1 & 3,8 \\
\hline Atemfrequenz $>25 / \mathrm{min}$ & 3,4 & 0,8 \\
\hline \multicolumn{3}{|l|}{ Lungenembolie } \\
\hline Wells-Score & 5,9 & 0,1 \\
\hline Geneva-Score & 5,8 & 0,4 \\
\hline Subjektive klinische Einschätzung (Kaderarzt/ärztin) & 8,0 & 0,1 \\
\hline Subjektive klinische Einschätzung (Assistent/in) & 4,5 & 0,4 \\
\hline \multicolumn{3}{|l|}{ Notwendigkeit einer mechanischen Beatmung bei COPD } \\
\hline Asynchrone Atmung & 3,5 & 0,3 \\
\hline \multicolumn{3}{|l|}{ Lungenkarzinom } \\
\hline "Clubbing" & 3,9 & 0,7 \\
\hline \multicolumn{3}{|l|}{ Vorliegen einer Linksherzinsuffizienz bei akuter Dyspnoe } \\
\hline Anamnese eines Myokardinfarkts & 3,1 & 0,7 \\
\hline Paroxysmale nächtliche Dyspnoe & 2,6 & 0,7 \\
\hline Orthopnoe & 2,2 & 0,7 \\
\hline 3. Herzton & 11 & 0,9 \\
\hline Halsvenenstauung & 5,1 & 0,7 \\
\hline Hepatojugulärer Reflux & 6,4 & 0,8 \\
\hline Rasselgeräusche & 2,8 & 0,5 \\
\hline Herzgeräusch & 2,6 & 0,4 \\
\hline Beinödeme & 2,1 & 0,6 \\
\hline Klinische Gesamtbeurteilung & 3,1 & 0,09 \\
\hline $\mathrm{BNP}>200 \mathrm{pg} / \mathrm{ml}$ & 3,7 & 0,11 \\
\hline
\end{tabular}

Anamnese und Unersuchung haben auch eine wichtige prognostische Aussagekraft
Anamnese und Untersuchung haben auch eine wichtige prognostische Aussagekraft, so z. B. eine asynchrone Atmung bei einer Exazerbation einer COPD [18] oder der bestens bekannte CRB-65Score bei einer Pneumonie [19].

\section{Fazit für die Praxis}

- Auch heute noch können 80\% aller Diagnosen mittels Anamnese und klinischer Untersuchung gestellt werden. Weitere, zumeist apparative Tests dienen meist nur noch der Bestätigung der Diagnose.

- Eine kompetente Anamnese und klinische Untersuchung machen nicht selten teure und/oder zeitaufwendige Explorationen unnötig. 


\title{
- Die aufgrund des klinischen Eindrucks gewonnene subjektive Einschätzung der Vortest- Wahrscheinlichkeit für das Vorliegen einer Krankheit ist eine unabdingbare Voraussetzung für die Bewertung weiterer Testresultate.
}

\section{Korrespondenzadresse}

\author{
Prof. Dr. R. Speich
}

Universitätsspital Zürich

Rämistr. 100, 8091 Zürich

rudolf.speich@usz.ch

Interessenkonflikt. Der korrespondierende Autor gibt an, dass kein Interessenkonflikt besteht.

\section{Literatur}

1. Fred HL (2005) Hyposkillia: deficiency of clinical skills. Tex Heart Inst J 32:255-257

2. ATS Committee on Proficiency Standards for Clinical Pulmonary Function Laboratories (2002) ATS statement: guidelines for the six-minute walk test. Am J Respir Crit Care Med 166:111-117

3. Puhan MA, Guyatt GH, Goldstein $R$ et al (2007) Relative responsiveness of the Chronic Respiratory Questionnaire, St. Georges Respiratory Questionnaire and four other healthrelated quality of life instruments for patients with chronic lung disease. Respir Med 101:308-316

4. Cenedese E, Speich R, Dorschner L et al (2006) Measurement of quality of life in pulmonary hypertension and its significance. Eur Respir J 28:808815

5. Puhan MA, Behnke M, Frey M et al (2004) Self-administration and interviewer-administration of the German Chronic Respiratory Questionnaire: instrument development and assessment of validity and reliability in two randomised studies. Health Qual Life Outcomes 2:1

6. Warschburger P, Busch S, Bauer CP et al (2004) Health-related quality of life in children and adolescents with asthma: results from the ESTAR Study. J Asthma 41:463-470

7. McKenna S, Doughty N, Meads D et al (2006) The Cambridge Pulmonary Hypertension Outcome Review (CAMPHOR): a measure of health-related quality of life and quality of life for patients with pulmonary hypertension. Qual Life Res 15:103-115

8. Johns MW (1991) A new method for measuring daytime sleepiness: the Epworth Sleepiness Scale. Sleep 14:540-545
9. Straus SE, McAlister FA, Sackett DL, Deeks JJ (2000) The accuracy of patient history, wheezing, and laryngeal measurements in diagnosing obstructive airway disease. CARE-COAD1 Group. Clinical Assessment of the Reliability of the Examination-Chronic Obstructive Airways Disease. JAMA 283:1853-1857

10. Bettencourt PE, Del Bono EA, Spiegelman D et al (1994) Clinical utility of chest auscultation in common pulmonary diseases. Am J Respir Crit Care Med 150:1291-1297

11. Geddes DM, Corrin B, Brewerton DA et al (1977) Progressive airway obliteration in adults and its association with rheumatoid disease. Q J Med 46:427-444

12. Loudon R, Murphy RL Jr (1984) Lung sounds. Am Rev Respir Dis 130:663673

13. Pasterkamp H, Kraman SS, Wodicka GR (1997) Respiratory sounds. Advances beyond the stethoscope. Am J Respir Crit Care Med 156:974-987

14. Paley L, Zornitzki T, Cohen J et al (2011) Utility of clinical examination in the diagnosis of emergency department patients admitted to the department of medicine of an academic hospital. Arch Intern Med 171:1394-1396

15. Dijk N van, Boer KR, Colman N et al (2008) High diagnostic yield and accuracy of history, physical examination, and ECG in patients with transient loss of consciousness in FAST: the Fainting Assessment study. J Cardiovasc Electrophysiol 19:48-55

16. Baron-Esquivias G, Martinez-Alday J, Martin A et al (2010) Epidemiological characteristics and diagnostic approach in patients admitted to the emergency room for transient loss of consciousness: Group for Syncope Study in the Emergency Room (GESINUR) study. Europace 12:869-876
17. Courtney DM, Kline JA, Kabrhel C et al (2010) Clinical features from the history and physical examination that predict the presence or absence of pulmonary embolism in symptomatic emergency department patients: results of a prospective, multicenter study. Ann Emerg Med 55:307-315 e301

18. Ashutosh $\mathrm{K}$, Gilbert R, Auchincloss $\mathrm{JH}$ Jr, Peppi D (1975) Asynchronous breathing movements in patients with chronic obstructive pulmonary disease. Chest 67:553-557

19. Capelastegui A, Espana PP, Quintana JM et al (2006) Validation of a predictive rule for the management of community-acquired pneumonia. Eur Respir J 27:151-157

20. Morgan WL, Engel GL (1977) Der klinische Zugang zum Patienten: Anamnese und Körperuntersuchung. Huber, Bern

21. Speich R (1997) Der diagnostische Prozess in der Inneren Medizin: Entscheidungsanalyse oder Intuition? Schweiz Med Wochenschr 127:12631279 
Bitte beachten Sie:

- Antwortmöglichkeit nur online unter: CME.springer.de

- Die Frage-Antwort-Kombinationen werden online individuell zusammengestellt.

- Es ist immer nur eine Antwort möglich.

Ein 32-jähriger Patient sucht Sie wegen seit einigen Wochen progredienter Anstrengungsdyspnoe auf. Die Lungenauskultation ist normal. Im Thoraxröntgenbild zeigen sich aber ausgedehnte bilaterale azinäre Infiltrate. Diese Trias ist pathognomonisch für:

$\square$ Eine Hypersensitivitätspneumonitis

$\square$ Eine Legionellen-Pneumonie

$\square$ Eine subakute Herzinsuffizienz

$\square$ Eine Pneumozystis-Pneumonie

$\square$ Eine Sarkoidose Stadium 2

Ein 56-jähriger Patient klagt über Anstrengungsdyspnoe, die in den letzten 3 bis 4 Monaten langsam zugenommen habe. Es fallen Ihnen deutliche Trommelschlegelfinger auf. Bei der Untersuchung der Lungen finden Sie perkutorisch eine hohe Zwerchfellgrenze etwa beim 8. Thoraxwirbel und feinblasige, klingende Rasselgeräusche. Diese Trias ist pathognomonisch für:

$\square$ Eine Hypersensitivitätspneumonitis

$\square$ Eine idiopathische Lungenfibrose

$\square$ Eine Herzinsuffizienz mit bilateralen Pleuraergüssen

$\square$ Eine Pneumozystis-Pneumonie

$\square$ Eine Sarkoidose Stadium 2

Eine 18-jährige Frau sucht sie wegen seit Langem bestehender chronischer Bronchitis auf. Es fallen Ihnen deutliche Trommelschlegelfinger auf. Bei der Untersuchung der Lungen finden Sie perkutorisch eine tiefe Zwerchfellgrenze etwa beim 12. Thoraxwirbel und grobblasige Rasselgeräusche. Diese Trias ist pathognomonisch für:

Eine Hypersensitivitätspneumonitis

$\square$ Eine Langerhans-Zell-Histiozytose

$\square$ Eine zystische Fibrose

$\square$ Eine Lymphangioleiomyomatose

Einen a-1-Antitrypsinmangel

\section{CME-Fragebogen kostenfreie Teilnahme für Abonnenten}

Eine 43-jährige Patientin klagt über Anstrengungsdyspnoe, die in den letzten 3 bis 4 Monaten langsam zugenommen habe. Bei der Auskultation hören Sie ein inspiratorisches Quietschen. Zu Ihrer Überraschung sind aber die Spirometrie und das Thoraxröntgenbild normal. Diese Trias ist pathognomonisch für:

Eine Bronchiolitis obliterans

$\square$ Eine Lymphangioleiomyomatose

$\square$ Eine Langerhans-Zell-Histiozytose

$\square$ Eine chronische Aspiration bei gastroösophagealem Reflux

Eine Sarkoidose Stadium 1

Ein 24-jähriger Patient kommt in Ihre Praxis und beschreibt die typischen Symptome eines Atemwegsinfektes. Was spricht am stärksten gegen das Vorliegen einer bakteriellen Pneumonie?

$\square$ Eine Atemfrequenz unter $20 / \mathrm{min}$

$\square$ Fehlendes Fieber

$\square$ Kein Schüttelfrost

$\square$ Vorbestehendes Asthma bronchiale

$\square$ Das junge Alter

Ein 72-jähriger Patient kommt in die Notfallstation wegen akuter Dyspnoe. Aufgrund Ihrer subjektiven Einschätzung ist die Chance für das Vorliegen einer akuten Lungenembolie hoch, d. h. etwa 4:1. Ihre erste Maßnahme, vorausgesetzt, es liegt kein hohes Blutungsrisiko vor, ist: Blutentnahme zur Bestimmung der DDimere

$\square$ Niedrigmolekulares Heparin subkutan

$\square$ Computertomogramm des Thorax

$\square$ Ultraschall der Beinvenen

Eine Magnetresonanztomographie
Eine 65-jährige Patientin klagt über Atemnot, die in den letzten 3 bis 4 Monaten langsam zugenommen habe. Im Thoraxröntgenbild finden Sie bilaterale interstitiell-azinäre Infiltrate und eine Kardiomegalie. Was spricht am stärksten für das Vorliegen einer Linksherzinsuffizienz?

Atemnot bei Anstrengung

Frühmorgendliche Dyspnoe mit Schweißausbruch

Beidseitige Beinödeme

Ein lautes Systolikum über dem Erb-Punkt

Bilaterale Rasselgeräusche

Ein 69-jähriger Patient mit bekannter COPD kommt in die Notfallstation wegen einer schweren akuten Exazerbation. Bei welchem Befund sollten Sie sich nach einem Platz auf einer Intensiv- oder Intermediate-Care-Station umschauen?

$\square$ Tachypnoe $>24 / \mathrm{min}$

$\square$ Asynchrone Atmung

$\square \mathrm{p}_{\mathrm{a}} \mathrm{O}_{2}<6,0 \mathrm{kPa}(<45 \mathrm{mmHg})$

$\mathrm{p}_{\mathrm{a}} \mathrm{CO}_{2}>8,0 \mathrm{kPa}(>80 \mathrm{mmHg})$

$\mathrm{FEV}_{1}<1,0 \mathrm{I}$

Sie haben einen 60-jährigen Patienten mit einem Nikotinabusus von 50 "pack years". Sie vermuten eine COPD, ihr Spirometer ist aber defekt. Welcher klinische Parameter erlaubt Ihnen, auch so eine COPD „mit hoher Wahrscheinlichkeit" zu diagnostizieren?

Giemen

$\square$ Rasselgeräusche

$\square$ Minimale laryngeale Distanz

$\square$ Maximale laryngeale Distanz

Forcierte exspiratorische Zeit von $6 \mathrm{~s}$ 
? Sie betreuen eine 40-jährige Frau mit akuter Atemnot. Aufgrund der Klinik schätzen Sie die Wahrscheinlichkeit für eine Lungenembolie um die $\mathbf{5 0} \%$. Sie machen einen D-Dimer-Test, von dem Sie wissen, dass er eine Sensitivität von $\mathbf{9 8 \%}$ und eine Spezifität von $\mathbf{5 0} \%$ hat. Das Testresultat ist negativ. Welches ist ungefähr die Wahrscheinlichkeit, dass die Frau eine Lungenembolie hat?

$\square 10 \%$

$\square 8 \%$

$\square 6 \%$

$\square 4 \%$

口 $2 \%$

Diese Fortbildungseinheit ist 12 Monate auf CME.springer.de verfügbar.

Den genauen Einsendeschluss erfahren Sie unter CME.springer.de

\section{CME.springer.de}

\section{Springer Medizin}

\section{CME.Tickets: Zertifizierte Fortbildung für alle!}

Auf CME.springer.de stehen Ihnen über 300 jährlich wechselnde Fortbildungseinheiten aus über 30 Bereichen der Medizin zu Verfügung. Punkten Sie jetzt online auf CME.springer.de!

1. Teilnahmemöglichkeiten: - kostenfrei im Rahmen des jeweiligen Zeitschriftenabonnements

- individuelle Teilnahme durch den Erwerb von CME.Tickets auf CME.springer.de.

\section{CME.Ticket erwerben}

Auf CME.springer.de haben Sie 2 Möglichkeiten CME.Tickets zu erwerben:

- CME.Ticket bestellen: Klicken Sie auf Bestellen > CME.Ticket und erwerben Sie hier Ihre individuelle Teilnahmemöglichkeit

\begin{abstract}
- CME.Ticket im Beitragsumfeld kaufen und einlösen: Sobald Sie an einem Beitrag außerhalb Ihres Abonnements teilnehmen möchten, erscheint der Hinweis CME.Ticket bestellen. Nach dem Erwerb des CME.Tickets können Sie an der gewünschten Fortbildungseinheit teilnehmen.
\end{abstract}

\section{Punkten Sie online!}

Bei Fragen hilft Ihnen unser Helpdesk gerne weiter: CME@springer.com

CME.springer.de 


\section{Hier steht eine Anzeige.}

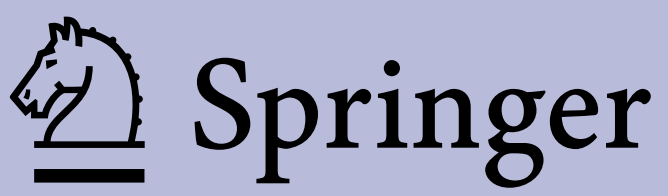

